\title{
Supplementary Material \\ Aminoacetylation Reaction Catalyzed by Leucyl-tRNA Synthetase Operates via a Self-Assisted Mechanism Using a Conserved Residue and the Aminoacyl Substrate.
}

\author{
Alexey Aleksandrov ${ }^{1 *}$, Andrés Palencia², Stephen Cusack $^{3}$, and Martin \\ Field ${ }^{4}$ \\ ${ }^{1}$ Laboratoire de Biochimie (CNRS UMR7654), Department of Biology, \\ Ecole Polytechnique, 91128 Palaiseau, France. \\ ${ }^{2}$ Institut Albert Bonniot, Team Host-pathogen interactions \\ immunity to infection, INSERM U1209, 38000 Grenoble, France. \\ ${ }^{3}$ European Molecular Biology Laboratory (EMBL), \\ Grenoble Outstation and Unit of Virus Host-Cell Interactions, \\ University of Grenoble-EMBL-CNRS, Grenoble, France. ${ }^{4}$ Dynamo Team, \\ DYNAMOP Group, \\ UMR 5075, Université Grenoble 1, CNRS, CEA, \\ Institut de Biologie Structurale, \\ 71 Avenue des Martyrs, CS 10090, 38044 Grenoble Cedex 9, France. \\ *Email: alexey.aleksandrov@polytechnique.fr
}

\section{Force field determination for leucyl-adenylate and the toxic moiety from Agrocin 84}

As a part of this study, we report a force field model for leucyl-adenylate (Lad) and the toxic moiety of Agrocin 84 (Tm84); see Fig. 1. The force field is developed so as to be compatible with the CHARMM27 force field for proteins and nucleic acids $[1,2]$, and with the TIP3P water model [3].

Structures were taken from the PDB. Atomic charges were derived from a supermolecule, ab initio approach, and Lennard-Jones parameters were adopted from the CHARMM27 force field $[1,2,4]$. We considered the ab initio energies and geometries of a water molecule interacting with each ligand at a few, selected positions. This supermolecule approach is known to give a good balance between solute-water and water-water interactions in the force field [1, 2]. Good agreement was obtained between the ab initio and force field data (rms deviation for the energies of $0.2 \mathrm{kcal} / \mathrm{mol}$ ). 

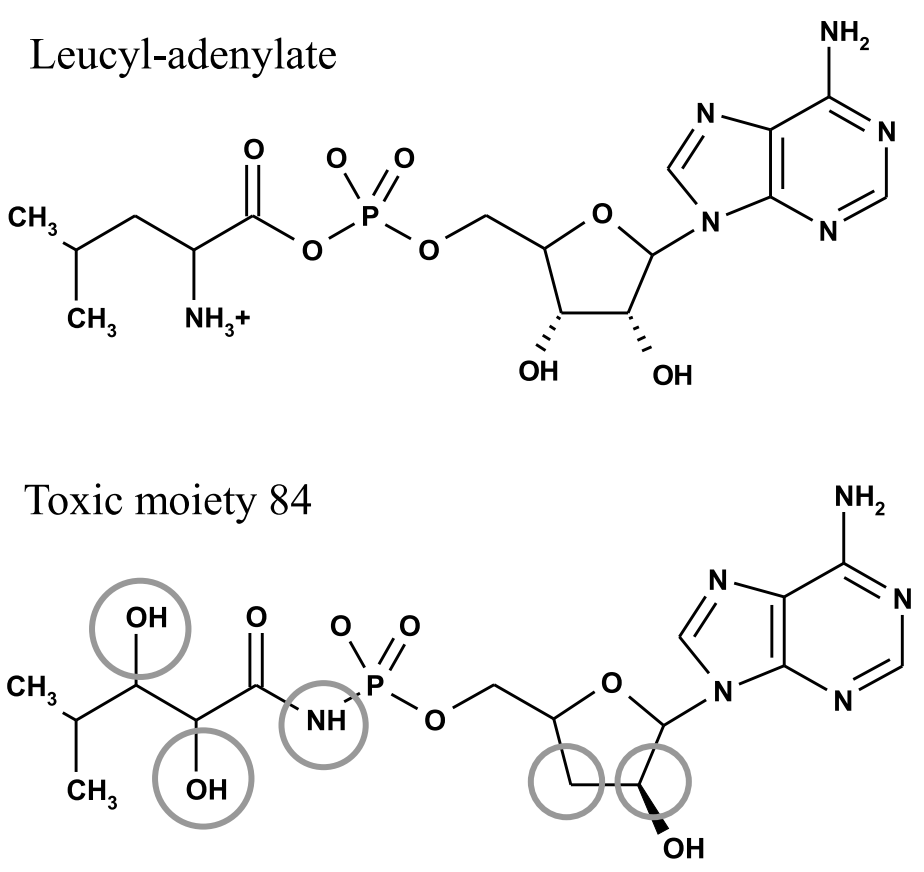

Figure S1: 2D view of leucyl-adenylate and the toxic moiety 84. Positions that are modified in Tm84, relative to leucyl-adenylate, are circled.

The model also reproduces well the ab initio geometry and flexibility of each ligand, and the interactions between ligands and protein. The model should thus be suitable to investigate the interactions of leucyl-tRNA and its analog with proteins.

\section{Optimization of the intermolecular force field parameters}

We adopt a force field of the CHARMM27 form [1, 2, 4], which is used to simulate complexes involving ligands, protein and RNA in aqueous solution. The intermolecular energy terms are Lennard-Jones and Coulomb terms. In accord with the development of the CHARMM27 force field, we rely on supermolecule, quantum chemical calculations on complexes between a model compound and a single water molecule. The position of the water is varied around the compound. The geometry of each model compound was optimized at the HF/6-31G(d) level. For the force field calculations, the water geometry was taken from the TIP3P model [3].

Each supermolecule structure was optimized at the $\mathrm{HF} / 6-31 \mathrm{G}(\mathrm{d})$ level by varying the interaction distance and a single angle, to find the local minimum for the water position. From the resulting optimal structure, the interaction energy was calculated. No correction for basis set superposition error was made. The ab initio interaction 
energies were scaled by a factor of 1.1, and to compensate for overestimated interaction distances with the Hartree-Fock model (due to neglected electron correlation), the ab initio interaction distances were reduced by $0.2 \AA[1]$.

The force field parameters were then adjusted to reproduce these "corrected" ab initio interaction energies and water positions. Ab-initio dipole moments were also included in the optimization. Initial partial charges were obtained from a Mulliken population analysis of the $\mathrm{HF} / 6-31 \mathrm{G}(\mathrm{d})$ wavefunction and were compared with charges of similar molecules for which CHARMM parameters are known. Lennard-Jones parameters were adapted from the CHARMM27 force field for similar chemical groups.

A few water positions were considered for each model compound. For leucyladenylate, we parametrized two model compounds: acetyl-methylphosphate and acetylmethylphosphoramidate. We optimized the interaction distances and the (corrected) ab initio data were then fitted by varying manually the model ccompound charges. This involved reoptimizing the compound-water distance after each parameter change. Figure shows the final charges for each compound, whereas tables S3, S4, and S5 summarize the results obtained for water-ligand interactions and dipole moments.

(A)

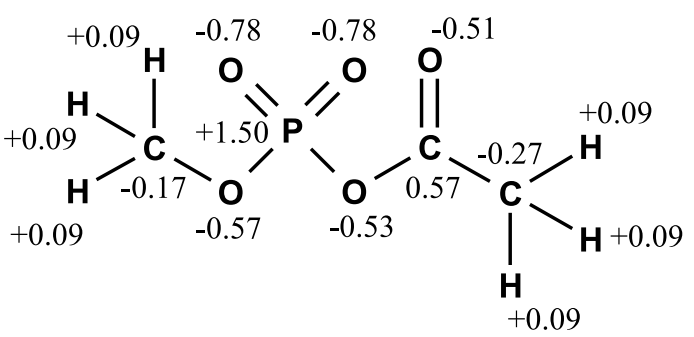

(B)

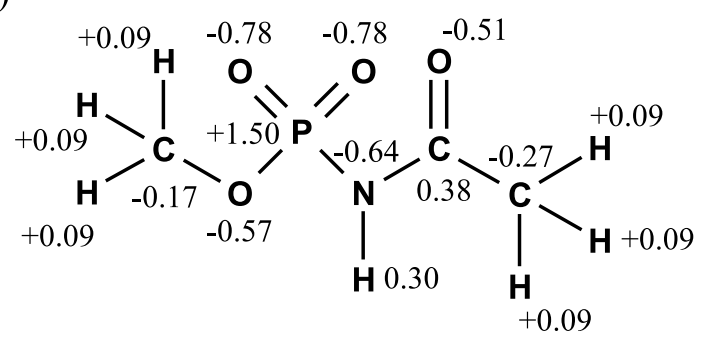

Figure S2: Charges developed in this work for (A) acetyl-methylphosphate and (B) acetyl-methylphosphoramidate. 


\section{Optimization of the intramolecular force field parameters}

In a force field treatment, the intramolecular geometry is mainly determined by the minimum energy values of the bond length and bond angle terms, and by the phase and multiplicity of the dihedrals. These parameters were optimized by fitting to the structures from the ab initio optimizations. At each parameter optimization step, the structure was minimized with the force field model, using a Powell conjugate gradient algorithm, stopping when the rms energy gradient reached $10^{-6} \mathrm{kcal} / \mathrm{mol} / \AA$. The quality of the parameters was measured by the sum of the rms deviations from the ab initio structure. The most difficult part was the adjustment of the dihedral force constants and phases. Starting guesses were taken from the CHARMM [1] force field for related, small molecule fragments.

The minimum energy bond lengths, bond angles and torsion angles were taken directly from the ab initio structures, optimized using the HF/6-31G(d) method. The rms deviation between the ab initio minimized structures and the force field minimized structures, after superposition of the molecules, was at most $0.3 \AA$.

To characterize leucyl-adenylate and Tm84 flexibility, we examined two kinds of fluctuations: small, harmonic fluctuations, which involve the stiff bond and angle degrees of freedom, and the larger, lower-frequency fluctuations, which involve the softer dihedral angles. Fig. 3 illustrates the small, harmonic fluctuations for the two model compounds that we studied, acetyl-phosphate and acetyl-phosphoramidate. The figure shows the normal mode frequencies, along with the corresponding rms positional fluctuations of important atoms. For all these properties, agreement between the ab initio and force field results is very good.

Fig. 4 and 5 illustrates the softer dihedral fluctuations. Energy profiles are shown for two dihedral angles, which link the AMP and aminoacyl group, and also the analogues angles in Tm84. Again, agreement is very good between the force field and the ab initio profiles, not only for the energy wells, but also for the barriers between wells.

\section{Ligands protein interactions: molecular dynamics simulations}

We performed MD simulations of the Lad:LeuRS and Tm84:LeuRS complexes for 5 nanoseconds each. Details of the MD simulations are given in Methods in the main manuscript.

We computed rms deviations from the crystal structure, averaging over the last 1 ns of the 5 ns simulations (table S1). By superimposing the leucyl-adenylate on the crystal structure, we obtain intramolecular leucyl-adenylate deformations of less than 

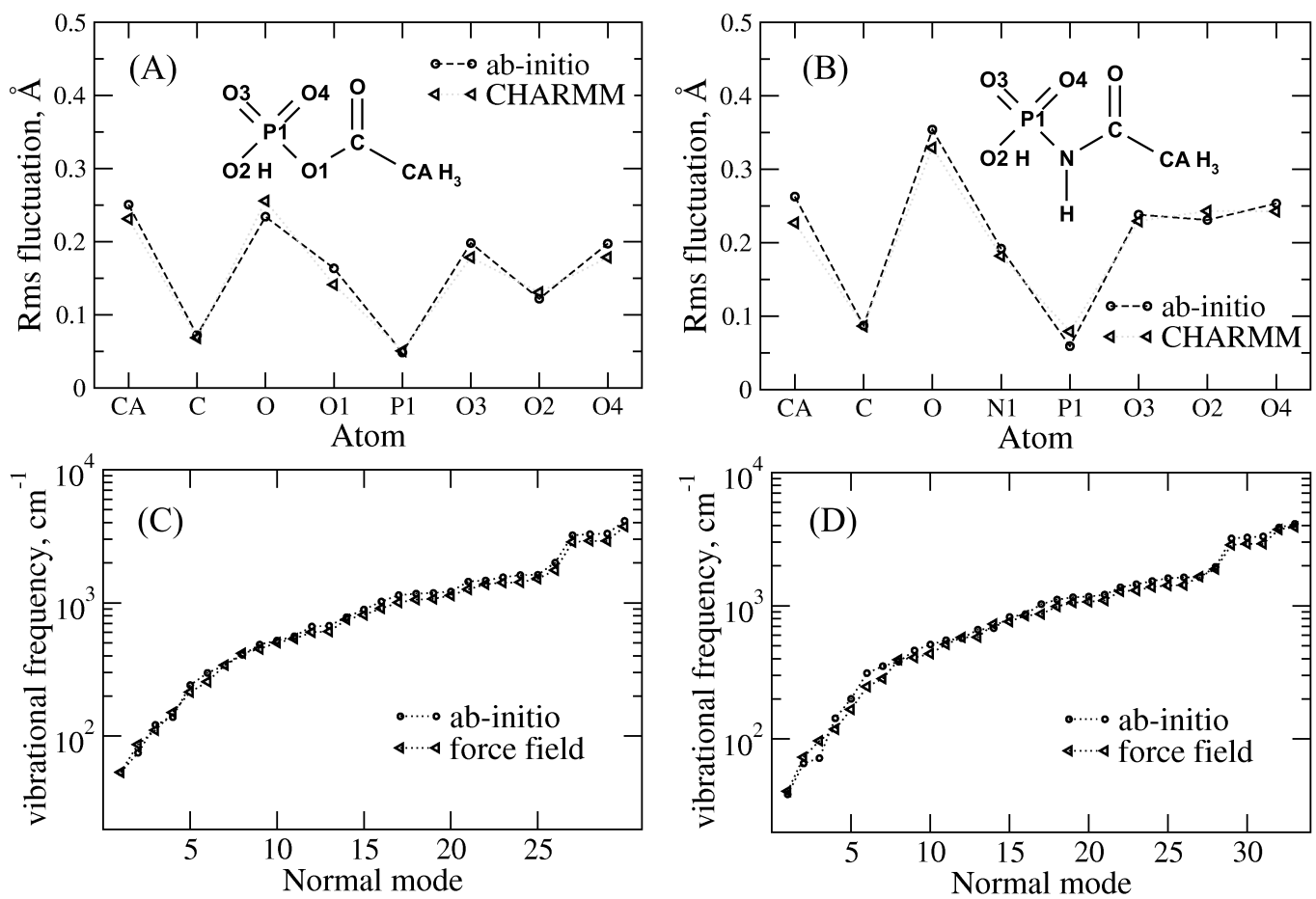

Figure S3: Rms positional fluctuations (A) and (B), and normal mode frequencies (C) and (D), for the two fragments shown in the insets: acetyl-phosphate and acetylphosphoramidate.

$0.5 \AA$. For Tm84 in the 84:LeuRS simulation we get $0.1 \AA$ rms deviation. Superimposing the protein backbone on the crystal structure, we obtain similar rms deviations of 0.7 $\AA$ for both Lad and Tm84. Likewise, the protein backbone rms deviations are both approximately $1.0 \AA$ for LeuRS in the Lad:LeuRS and Tm84:LeuRS simulations. The interactions between Lad, Tm84 and protein are also well-reproduced (table S2). 


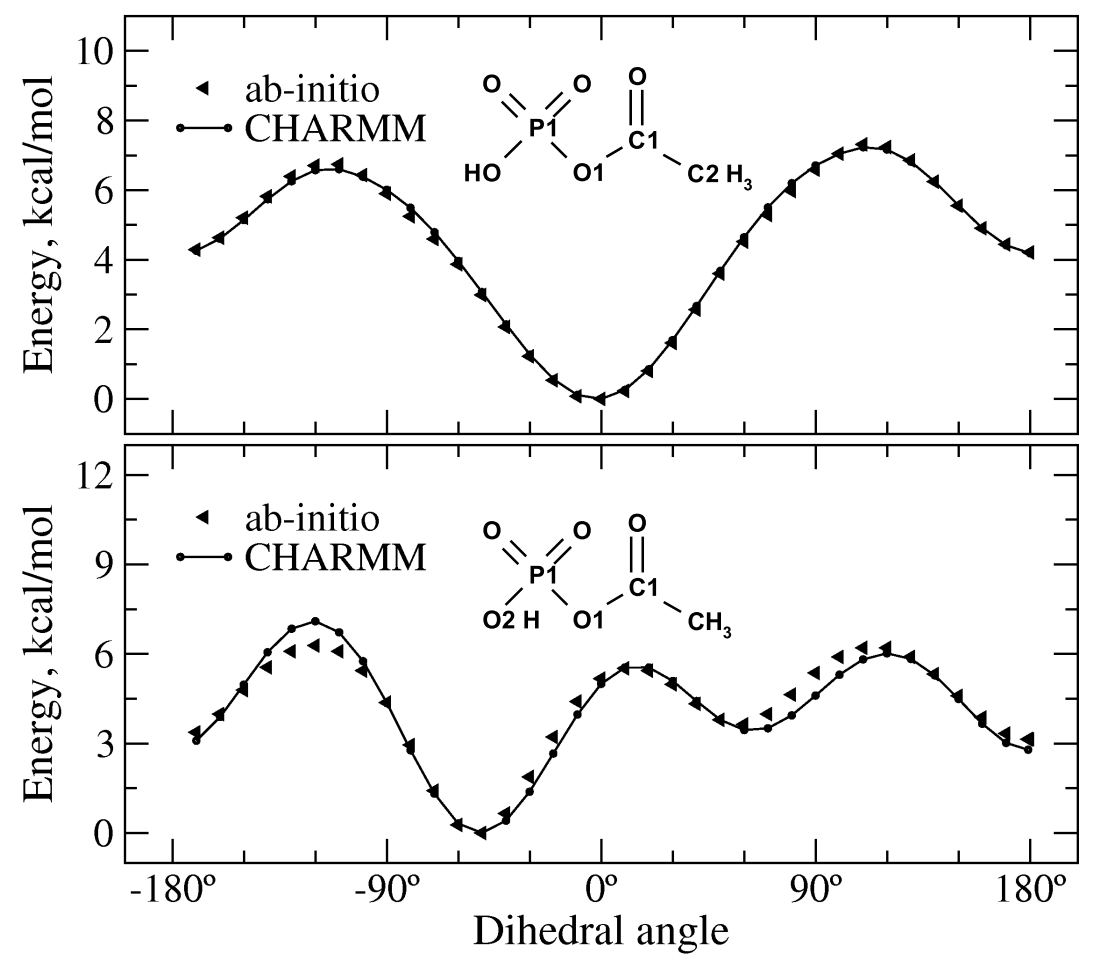

Figure S4: Comparing ab initio and force field energies for two dihedral angles in acetyl-phosphate. The dihedral angles are defined by the atoms labelled in each inset, namely C2-C1-O1-P1 and C1-O1-P1-O2. In each panel, the solid line corresponds to force field energies.

Table S1: Rms deviations $(\AA)$ for leucyl-tRNA synthetase complexes

\begin{tabular}{cccc}
\hline \hline ligand & ligand $^{a}$ & ligand $^{b} /$ backbone & backbone \\
\hline Lad & 0.36 & 0.59 & 0.64 \\
Tm84 & 0.34 & 0.45 & 0.63 \\
\hline \hline
\end{tabular}

Rms deviations between the MD and crystal structures. ${ }^{a}$ After superimposing the MD ligand on the ligand in the crystal structure. ${ }^{b}$ After superimposing the protein backbone on the crystal structure. Only non-hydrogen atoms are considered. 


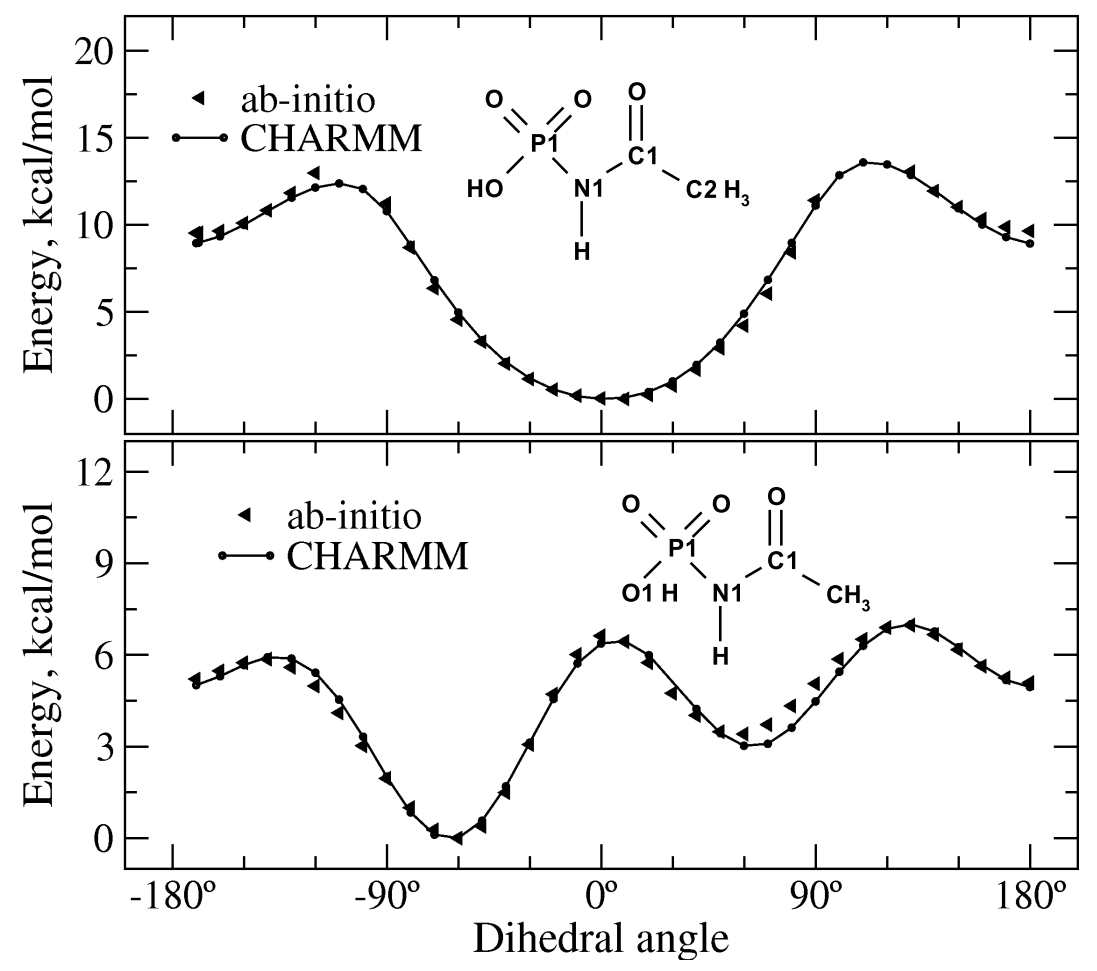

Figure S5: Comparing ab initio and force field energies for two dihedral angles in acetylphosphoramidate. The dihedral angles are defined by the atoms labelled in each inset, namely C2-C1-N1-P1 and C1-N1-P1-O1. In each panel, the solid line corresponds to force field energies.

Table S2: Selected distances $(\AA)$ between atoms of leucyl-adenylate and Tm84 and leucyl-tRNA synthetase

\begin{tabular}{|c|c|c|}
\hline $\begin{array}{l}\text { atom } \\
\text { pair }\end{array}$ & $\begin{array}{c}\text { MD } \\
\text { simulation }\end{array}$ & $\begin{array}{c}{ }^{a} \mathrm{X} \text {-ray } \\
\text { structure }\end{array}$ \\
\hline $\mathrm{O}_{L 41} \mathrm{~N}_{L a d}$ & 2.7 & 2.8 \\
\hline $\mathrm{O} \delta_{D 80} \mathrm{~N}_{L a d}$ & 2.6 & 2.7 \\
\hline $\mathrm{N} \epsilon_{H 533} \mathrm{O}_{L a d}$ & 2.8 & 3.1 \\
\hline $\mathrm{N}_{Y 43} \mathrm{O}_{1 \mathrm{P}} \mathrm{P}_{\text {Lad }}$ & 2.9 & 2.8 \\
\hline $\mathrm{O}_{L 41} \mathrm{OH}_{T m 84}$ & 3.1 & 3.2 \\
\hline $\mathrm{N} \epsilon_{H 533} \mathrm{O}_{T m 84}$ & 2.9 & 2.9 \\
\hline $\mathrm{N}_{Y 43} \mathrm{O}_{1 \mathrm{P}} \mathrm{P}_{T m 84}$ & 2.8 & 2.8 \\
\hline $\begin{array}{l}\text { LeuRS atoms } \\
\text { the amino acid } \\
{ }^{a} \text { PDB entries } 4\end{array}$ & ft) are & $\begin{array}{l}\text { lled by } \\
\text { belong. } \\
\mathrm{Z}[6] \text {. }\end{array}$ \\
\hline
\end{tabular}


Table S3: Interactions between a probe water and selected acetyl-methylphosphate sites

\begin{tabular}{cccc}
\hline \hline \multicolumn{4}{c}{ ab initio/force field results } \\
probe & energy & distance & angle \\
site & $(\mathrm{kcal} / \mathrm{mol})$ & $(\AA)$ & $\left({ }^{\circ}\right)$ \\
\hline OC & $-8.03 /-7.78$ & $1.90 / 1.76$ & 0.0 \\
OC & $-8.07 /-8.07$ & $1.90 / 1.76$ & 60.0 \\
OC & $-8.57 /-8.82$ & $1.90 / 1.75$ & 120.0 \\
OC & $-9.06 /-9.29$ & $1.90 / 1.75$ & 180.0 \\
OC & $-8.83 /-8.96$ & $1.90 / 1.75$ & 240.0 \\
OC & $-8.30 /-8.20$ & $1.90 / 1.76$ & 300.0 \\
O1C & $-9.94 /-9.98$ & $1.98 / 1.91$ & 0.0 \\
O1C & $-8.68 /-8.69$ & $2.04 / 1.96$ & 60.0 \\
O1C & $-7.49 /-7.44$ & $2.08 / 1.98$ & 120.0 \\
O1C & $-7.76 /-7.39$ & $2.03 / 1.96$ & 180.0 \\
O1C & $-6.69 /-6.87$ & $2.11 / 1.99$ & 240.0 \\
O1C & $-7.49 /-7.96$ & $2.08 / 1.97$ & 300.0 \\
\hline \hline
\end{tabular}


Table S4: Interactions between a probe water and selected acetylmethylphosphoramidate sites

\begin{tabular}{cccc}
\hline \hline \multirow{4}{*}{$\begin{array}{c}\text { ab initio/force field results } \\
\text { probe } \\
\text { site }\end{array}$} & $\begin{array}{c}\text { encal/mol }) \\
\text { distance }\end{array}$ & $\begin{array}{c}\text { angle } \\
\AA\end{array}$ & $\left(^{\circ}\right)$ \\
\hline OC & $-8.95 /-8.79$ & $1.90 / 1.74$ & 0.0 \\
OC & $-8.87 /-8.87$ & $1.90 / 1.74$ & 60.0 \\
OC & $-9.03 /-9.14$ & $1.90 / 1.74$ & 120.0 \\
OC & $-9.22 /-9.34$ & $1.90 / 1.74$ & 180.0 \\
OC & $-9.26 /-9.30$ & $1.90 / 1.74$ & 240.0 \\
OC & $-9.10 /-9.03$ & $1.90 / 1.74$ & 300.0 \\
NC & $-5.31 /-5.45$ & $2.60 / 2.42$ & 0.0 \\
NC & $-7.26 /-7.43$ & $2.37 / 2.27$ & 60.0 \\
NC & $-5.31 /-6.30$ & $2.55 / 2.32$ & 120.0 \\
NC & $-3.43 /-4.44$ & $2.86 / 2.43$ & 180.0 \\
NC & $-3.05 /-3.50$ & $2.88 / 2.52$ & 240.0 \\
NC & $-3.36 /-3.58$ & $2.86 / 2.61$ & 300.0 \\
\hline \hline
\end{tabular}

Table S5: Ab initio and empirical dipole moments

\begin{tabular}{cccc}
\hline \hline compound & \multicolumn{2}{c}{ dipole moment (debye) } & ${ }^{a}$ angle \\
& ab-initio & empirical & $\left(^{\circ}\right)$ \\
\hline acetyl-methylphosphate & 5.23 & 4.26 & 11.8 \\
acetyl-methylphosphoramidate & 4.69 & 3.39 & 0.7 \\
\hline \hline
\end{tabular}

${ }^{a}$ Angle between the ab initio and empirical dipole moment vectors.

\section{References}

[1] Mackerell, A. et al. J. Phys. Chem. B 1998, 102, 3586-3616.

[2] Mackerell, A.; Wiorkiewicz-Kuczera, J.; Karplus, M. J. Am. Chem. Soc. 1995, 117, 11946-11975. 
[3] Jorgensen, W.; Chandrasekar, J.; Madura, J.; Impey, R.; Klein, M. J. Chem. Phys. 1983, 79, 926-935.

[4] Foloppe, N.; MacKerell, A. J. Comp. Chem. 2000, 21, 86-104.

[5] Palencia, A.; Crepin, T.; Vu, M.; Lincecum, T. J.; Martinis, S.; Cusack, S. Nat. Struct. Biol. 2012, 19, 677-684.

[6] Chopra, S.; Palencia, A.; Virus, C.; Tripathy, A.; Temple, B.; VelazquezCampoy, A.; Cusack, S.; Reader, J. Nat. Commun. 2013, 1417. 